\title{
Performance of Space-Division Multiple-Access Systems Communicating over Nakagami Fading Channels
}

\author{
Lie-Liang Yang and Lajos Hanzo \\ Dept. of ECS, University of Southampton, SO17 1BJ, UK. \\ Tel: +44-23-8059 3125, Fax: +44-23-8059 4508 \\ Email: 1ly,lh@ecs.soton.ac.uk; http://www-mobile.ecs.soton.ac.uk
}

\begin{abstract}
Performance of a space-division multiple-access (SDMA) system is investigated, when the space-time block coded signals based on two transmit antennas are transmitted over Nakagami- $m$ fading channels. The receiver employs multiple receive antennas, each consisting of several antenna array elements. In order to gain insight into the multiuser interference resistance of SDMA systems, we consider the employment of a single-user detector. Our simulation results show that in SDMA systems the interfering signals arriving from directions different from that of the desired signal can be efficiently suppressed with the aid of the antenna arrays. However, due to the time-varying characteristics of wireless channels, aggressive interference may be imposed on the desired signal, when the interfering signals have a similar direction of arrival (DOA) to that of the desired signal. Therefore, in SDMA systems special emphasis must be put on the suppression of the multiuser interference aggravated by the effects of multipath fading.
\end{abstract}

\section{INTRODUCTION}

In wireless communications Multiple-Input-Multiple-Output (MIMO) systems equipped with multiple antennas at both the transmitter and receiver hold the promise of substantial spectral efficiency improvements relative to what is achieved at the current state-of-the-art [1], [2], [3]. Recently, MIMO systems have attracted intensive research interests in the context of both their theory and applications, as indicated by [4], [5] and the references in them. It is widely recognized that MIMO systems can be employed for achieving a high capacity [2], [3], a high diversity order [6], [7], for mitigating various types of interfering signals [8], and for supporting multiple users with the aid of space-division multiple-access (SDMA) [8]. The core principle behind the high spectral efficiency achieved by MIMO systems is that when communicating over a rich scattering propagation environment providing nearly independent transmission paths from each transmit antenna to each receive antenna, the multiple antennas employed by the transmitter and/or receiver are capable of providing extra degrees of freedom in the spatialdomain, in addition to the degrees of freedom provided in the conventionally exploited time-domain and frequency-domain. This increased degrees of freedom allows wireless systems to increase their capacity, to provide increased diversity, or to suppress the effects of interference, etc.

In this contribution the application of the MIMO principle in the context of SDMA is investigated. Specifically, the uplink communication scenario of Fig.1 is considered, where each mobile user employs two transmit antennas and the base-station
(BS) employs $M$ number of receive antenna arrays, each of which invokes $L$ number of array elements or sensors. At the transmitter side Alamouti's space-time coding scheme [6] is employed by the mobiles for encoding the transmitted symbols, in order to achieve transmit diversity gain. By contrast, at the BS the signals obtained by sampling the receive antenna arrays" output are linearly combined, in order to detect the information transmitted by the different SDMA users. In this contribution the employment of single-user detection schemes is studied, where we assume that the receiver has explicit knowledge of the desired user's parameters, including the desired user's direction-of-arrival (DOA) and the corresponding channel impulse responses (CIR) with respect to the $M$ number of receive antenna arrays. However, no knowledge of the other users" parameters is assumed.

The single-user detector considered in this contribution exhibits similarities to the conventional correlation or matchedfilter (MF) based receivers designed for direct-sequence codedivision multiple-access (DS-CDMA) systems [9]. Therefore, it is referred to as the spatial correlation receiver. In contrast to the popular Rayleigh fading channel model typically used for studying the performance of MIMO systems, in this contribution the performance of the SDMA system using the abovementioned spatial correlation receiver is studied, when communicating over Nakagami- $m$ fading channels. This generic channel model is used, since the Nakagami- $m$ distribution is a generalized distribution, which often gives the best fit to landmobile and indoor-mobile multipath propagation environments, as well as to scintillating ionospheric radio links. Furthermore, the Nakagami- $m$ distribution includes the Rayleigh distribution as a special case for $m=1$. It can also accurately approximate the Rician distributed fading, when $m>1$, with one-to-one mapping between the fading parameter $m$ and the Rician $K$ factor. Let us first consider the space-time signals received over the spatial channels considered.

\section{REPRESENTATION OF THE RECEIVED SignAL}

Fig. 1 shows the structure of a SDMA system, where each mobile transmitter employs two transmit antennas and the common receiver at the base station (BS) employs $M$ number of antenna arrays, each having $L$ elements. Note that in Fig. 1 the thin lines represent scalar variables, while the thick lines correspond to vector variables. As shown in Fig. 1, the adjacent data 


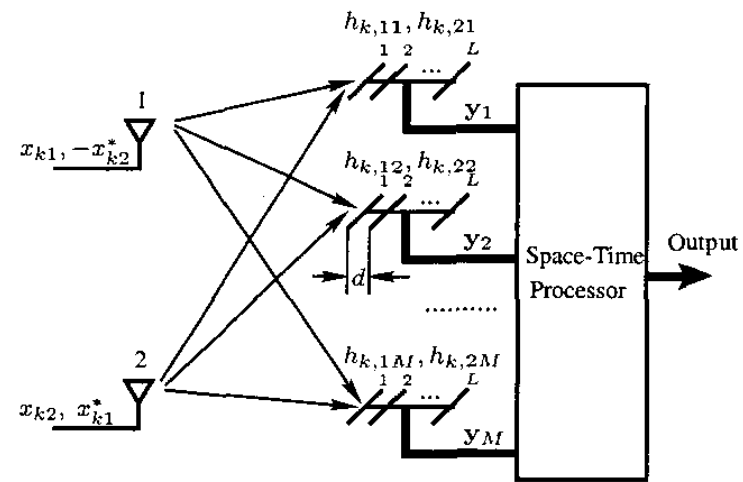

Fig. 1. Stylised schematic of the space-time block coding assisted transmitter. channel model as well as space-time receiver invoked for the SDMA system using two transmit antennus and $M$ receive antenna arrays.

symbols $x_{1}$ and $x_{2}$ are space-time block coded according to Alamouti's scheme [6] at the $k$ th user's mobile transmitter. The resultant space-time coded symbols are then transmitted during two consecutive symbol periods. In the first symbol period the symbol transmitted from antenna 1 is $x_{k 1}$ and the symbol transmitted from antenna 2 is $x_{k 2}$. By contrast, in the second symbol period the symbol transmitted from antenna 1 is $-x_{k 2}^{*}$ and the symbol transmitted from the antenna 2 is $x_{k 1}^{*}$, where the superscript ${ }^{*}$ denotes the complex conjugate.

Again, at the base-station there are $M$ number of receiver antenna arrays, as shown in Fig. 1 . We assume that all the $M$ different antenna arrays are located sufficiently far apart so that their received signals experience independent fading. As shown in Fig. 1, each of the $M$ antenna arrays consists of $L$ number of array elements, which are linearly correlated elements separated by a distance of $d$. The distance $d$ typically assumes a value of $\frac{\lambda}{2}$, namely half a wavelength. We assume that $K$ users are supported by the SDMA system. These users transmit their data synchronously over flat Nakagami fading channels. Furthermore, we assume that the complex fading envelope remains constant across two consecutive space-time coded symbol periods. Consequently, based on the above assumptions, the received signal vectors of the $m$ th BS antenna array during the two consecutive symbol periods can be expressed as

$$
\mathbf{y}_{m}=\sum_{k=1}^{K} \mathbf{r}_{k m}+\mathbf{n}_{\mathbf{m}}, m=1,2, \ldots, M
$$

where $\mathbf{y}_{m}$ is a $2 L$-dimensional vector representing the outputs of the $m$ th antenna array associated with a space-time coded period constituted by two consecutive symbol periods, and $\mathbf{n}_{m}$ is a $2 L$-dimensional vector representing the additive white Gaussian noise (AWGN). Each element of $\mathbf{n}_{m}$ is modelled as an independent identically distributed (iid) complex Gaussian random variable with zero mean and a power spectral density of $N_{0} / 2$ per dimension. Furthermore, in (1) $\mathbf{r}_{k m}$ represents the received signal component contributed by the $k$ th user, which can be expressed as

$$
\begin{aligned}
\mathbf{r}_{k m} & =\left[\begin{array}{l}
\mathbf{r}_{k, 1 m} \\
\mathbf{r}_{k, 2 m}^{*}
\end{array}\right] \\
& =\left[\begin{array}{ll}
\mathbf{d}_{k m} & \mathbf{0} \\
\mathbf{0} & \mathbf{d}_{k m}^{*}
\end{array}\right]\left[\begin{array}{ll}
h_{k, 1 m} & h_{k, 2 m} \\
h_{k, 2 m}^{*} & -h_{k, 1 m}^{*}
\end{array}\right]\left[\begin{array}{l}
x_{k 1} \\
x_{k 2}
\end{array}\right],
\end{aligned}
$$

where $\mathbf{r}_{k, 1 m}$ and $\mathbf{r}_{k, 2 m}$ represent the $m$ th array's outputs corresponding to the first and second space-time coded symbol periods, respectively, while $\mathbf{d}_{k m}$ represents the $L$-dimensional complex array vector in terms of the $k$ th user and the $m$ th antenna array. Since we assumed that the array elements are separated by half a wavelength, we have

$$
\mathbf{d}_{k m}=\left[1, e^{j \pi \sin \left(\psi_{k m}\right)}, \ldots, e^{j \pi(L-1) \sin \left(\psi_{k m}\right)}\right]^{T},
$$

where $\psi_{k m}$ represents the direction-of-arrival (DOA) corresponding to the $k$ th user and the $m$ th receive antenna array.

In (2) $h_{k, i m}=\left|h_{k, i m}\right| e^{j \theta_{k, i m}}$ represents the channel's impulse response in the context of the $k$ th user, the $i$ th, $i=$ 1,2 , transmit antenna and the $m$ th, $m=1,2, \ldots, M$, receive antenna array, where $\left|h_{k, i m}\right|$ represents the fading attenuation, while $\theta_{k, i m}$ represents the phase rotation imposed by the complex-valued Nakagami fading channel. More specifically, we assume that the fading attenuations $\left\{\left|h_{k, i m}\right|\right\}$ are independent Nakagami random variables having a probability density function (PDF) of

$$
p_{\left|h_{k, i m}\right|}(R)=\left(\frac{m}{\Omega}\right)^{m} \frac{2 R^{2 m-1}}{\Gamma(m)} \exp \left(-\frac{m R^{2}}{\Omega}\right),
$$

where $\Gamma(\cdot)$ is the gamma function, $m$ is the Nakagami- $m$ fading parameter, while $\Omega$ in (4) is the second moment of. $\left|h_{k, i m}\right|$, i.e., we have $\Omega=E\left[\left|h_{k, i m}\right|^{2}\right]$.

Let

$$
\begin{aligned}
\mathbf{D}_{k m} & =\left[\begin{array}{ll}
\mathbf{d}_{k m} & \mathbf{0} \\
\mathbf{0} & \mathbf{d}_{k m}^{*}
\end{array}\right], \\
\mathbf{H}_{k m} & =\left[\begin{array}{ll}
h_{k, 1 m} & h_{k, 2 m} \\
h_{k, 2 m}^{*} & -h_{k, 1 m}^{*}
\end{array}\right] \text { and } \mathbf{x}_{k}=\left[\begin{array}{l}
x_{k 1} \\
x_{k 2}
\end{array}\right] .
\end{aligned}
$$

Then, $\mathbf{r}_{k m}$ in (2) can be written as

$$
\mathbf{r}_{k m}=\mathbf{D}_{k m} \mathbf{H}_{k m} \mathbf{x}_{k}
$$

Furthermore, let $\mathbf{Y}=\left[\begin{array}{llll}\mathbf{y}_{1}^{T} & \mathbf{y}_{2}^{T} & \ldots & \mathbf{y}_{M}^{T}\end{array}\right]^{T}$. Then it can be readily shown that the received signal of (1) can be written as

$$
\mathbf{Y}=\sum_{k=1}^{K} \mathbf{D}_{k} \mathbf{H}_{k} \mathbf{x}_{k}+\mathbf{n}
$$

where $\mathbf{D}_{k}$ represents the $k$ th user's array-related matrix, which can be expressed as

$\mathbf{D}_{k}=\left[\begin{array}{cccc}\mathbf{D}_{k 1} & \mathbf{0} & \ldots & \mathbf{0} \\ \mathbf{0} & \mathbf{D}_{k 2} & \ldots & \mathbf{0} \\ \vdots & \vdots & \ddots & \vdots \\ \mathbf{0} & \mathbf{0} & \ldots & \mathbf{D}_{k M}\end{array}\right], k=1,2, \ldots, K$ 
while $\mathbf{H}_{k}$ is the channel-related matrix of user $k$, which can be expressed as

$$
\mathbf{H}_{k}=\left[\begin{array}{llll}
\mathbf{H}_{k 1}^{T} & \mathbf{H}_{k 2}^{T} & \ldots & \mathbf{H}_{k M}^{T}
\end{array}\right]^{T}, k=1,2, \ldots, K .
$$

Finally, in (7) $\mathbf{n}=\left[\begin{array}{llll}\mathbf{n}_{1}^{T} & \mathbf{n}_{2}^{T} & \ldots & \mathbf{n}_{M I}^{T}\end{array}\right]^{T}$ is a $2 M L$-dimensional AWGN vector, which is a zero-mean Gaussian vector having a covariance of

$$
\mathbf{R}_{n}=E\left[\mathbf{n n}^{H}\right]=N_{0} \mathbf{I}_{2 M L},
$$

where the superscript ${ }^{H}$ represents the conjugate transpose operation, while $\mathbf{I}_{2 M L}$ is the identity matrix of size $2 M L$.

Having characterized the SDMA signal, below the singleuser spatial correlation detector is investigated, where we assume that the receiver only employs the knowledge of the desired user's parameters. More explicitly, this knowledge includes both that of the array vector in (3) and that of the channel states in (5) in the context of the desired user. Specifically, let us assume that the first user is the user-of-interest, and assume that the receiver employs the knowledge of $\mathbf{d}_{11}, \mathbf{d}_{12}, \ldots, \mathbf{d}_{1 M}$ as well as the knowledge of $\mathbf{H}_{11}, \mathbf{H}_{12}, \ldots, \mathbf{H}_{1 M}$. The objective of the receiver is to detect $\mathbf{x}_{1}=\left[x_{11}, x_{12}\right]^{T}$ transmitted by the user-of-interest associated with the user-index of $k=1$.

\section{SPATIAL CoRrelation DETECTION OF SDMA SIGNALS}

In this section we consider the spatial correlation detection of SDMA signals. Firstly, the specific case of supporting $K=2$ users is considered. Then, the results obtained for $K=2$ users are extended to the general case of supporting $K>2$ number of users.

Alamouti's space-time code [6] has the property of $\mathbf{H}_{1 m}^{H} \mathbf{H}_{1 m}=\left(\left|h_{1,1 m}\right|^{2}+\left|h_{1,2 m}\right|^{2}\right) \mathbf{I}_{2}$. Consequently, in the specific case of $K=2$ users, the decision variables corresponding to the two symbols transmitted by the first user can be obtained by multiplying both sides of (7) with $\left(\mathbf{D}_{1} \mathbf{H}_{1}\right)^{H}$, which can be expressed as

$$
\mathbf{Z}_{1}=\left[\begin{array}{c}
Z_{11} \\
Z_{12}
\end{array}\right]=\left(\mathbf{D}_{1} \mathbf{H}_{1}\right)^{H} \mathbf{D H X}+\tilde{\mathbf{n}}
$$

where

$$
\tilde{\mathbf{n}}=\left(\mathbf{D}_{1} \mathbf{H}_{1}\right)^{H} \mathbf{n} .
$$

It can be shown that for a given set of fading values associated with user $1, \tilde{\mathbf{n}}$ is a zero-mean Gaussian vector having a covariance matrix expressed as

$$
\mathbf{R}_{\tilde{n}}=E\left[\tilde{\mathbf{n}} \tilde{\mathbf{n}}^{H}\right]=N_{0} L \sum_{m=1}^{M}\left(\left|h_{1,1 m}\right|^{2}+\left|h_{1,2 m}\right|^{2}\right) \mathbf{I}_{2} .
$$

Upon substituting $\mathbf{D}_{1}$ of (8), and $\mathbf{H}_{1}$ of (9) into (11), it can be shown that the expression of $\mathbf{Z}_{1}$ can be simplified to

$$
\mathbf{Z}_{1}=L \sum_{m=1}^{M}\left(\left|h_{1,1 m}\right|^{2}+\left|h_{1,2 m}\right|^{2}\right)\left(\mathbf{x}_{1}+\mathbf{R}_{12} \mathbf{x}_{2}\right)+\tilde{\mathbf{n}}
$$

where $\mathbf{R}_{12}$ represents the correlation matrix evaluated for user 1 and user 2 in terms of the array responses as well as channel states, which can be expressed as

$$
\begin{aligned}
\mathbf{R}_{12}= & \left(L \sum_{m=1}^{M}\left(\left|h_{1,1 m}\right|^{2}+\left|h_{1,2 m}\right|^{2}\right)\right)^{-1} \\
& \times \sum_{m=1}^{M} \mathbf{H}_{1 m}^{H} \mathbf{D}_{1 m}^{H} \mathbf{D}_{2 m} \mathbf{H}_{2 m} .
\end{aligned}
$$

Since the channel amplitudes are random variables, we infer from (15) that user 2 will impose interference on user 1, if there is a non-zero correlation between the array vector $\mathbf{D}_{1 m}$ of user 1 and the array vector $\mathbf{D}_{2 m}$ of user 2 for $m=1,2, \ldots, M$, i.e. if we have $\mathbf{D}_{1 m}^{H} \mathbf{D}_{2 m} \neq \mathbf{0}$ for any value of $m$, where $\mathbf{0}$ represents a matrix having elements of zero. By contrast, when these two users' signals are spatially orthogonal, i.e. when we have $\mathbf{D}_{1 m}^{H} \mathbf{D}_{2 \dot{m}}=\mathbf{0}$ for any value of $m$, then we have $\mathbf{R}_{12}=\mathbf{0}$. Consequently, in this scenario user 2 imposes no interference on user 1. Furthermore, upon substituting $\mathbf{D}_{1 m}, \mathbf{D}_{2 m}, \mathbf{H}_{1 m}$ and $\mathbf{H}_{2 m}$ from (5) into (15), $\mathbf{R}_{12}$ can be written as

$$
\begin{aligned}
& \mathbf{R}_{12}=(\left.\sum_{m=1}^{M}\left(\left|h_{1,1 m}\right|^{2}+\left|h_{1,2 m}\right|^{2}\right)\right)^{-1} . \\
& \times \sum_{m=1}^{M}\left[\begin{array}{l}
h_{1,1 m}^{*} h_{2,1 m} \rho_{12, m}+h_{1,2 m} h_{2,2 m}^{*} \rho_{12, m}^{*} \\
h_{1,2 m}^{*} h_{2,1 m} \rho_{12, m}-h_{1,1 m} h_{2,2 m}^{*} \rho_{12, m}^{*} \\
h_{1,1 m}^{*} h_{2,2 m} \rho_{12, m}-h_{1,2 m} h_{2,1 m}^{*} \rho_{12, m}^{*} \\
h_{1,2 m}^{*} h_{2,2 m} \rho_{12, m}+h_{1,1 m} h_{2,1 m}^{*} \rho_{12, m}^{*}
\end{array}\right],
\end{aligned}
$$

where $\rho_{12, m}$ represents the spatial correlation factor between the array vectors of user 1 and user 2 associated with the $m$ th antenna array. Explicitly, $\rho_{12, m}$ is given by

$$
\rho_{12, m}=\frac{1}{L} \mathbf{d}_{1 m}^{H} \mathbf{d}_{2 m} \text {. }
$$

Having considered the detection of $K=2$ users, the results can be readily extended to an arbitrary value $K$. By remembering that the received signal was expressed in (7), it can be readily shown that when $K$ number of users are supported, the decision variable $\mathbf{Z}_{1}$ corresponding to the symbols transmitted by the first user can be formulated as

$$
\begin{aligned}
\mathbf{Z}_{1}= & \left(\mathbf{D}_{1} \mathbf{H}_{1}\right)^{H} \sum_{k=1}^{K} \mathbf{D}_{k} \mathbf{H}_{k} \mathbf{x}_{k}+\tilde{\mathbf{n}} \\
= & L \sum_{m=1}^{M}\left(\left|h_{1,1 m}\right|^{2}+\left|h_{1,2 m}\right|^{2}\right) \\
& \times\left(\mathbf{x}_{1}+\sum_{k=2}^{K} \mathbf{R}_{1 k} \mathbf{x}_{k}\right)+\tilde{\mathbf{n}}
\end{aligned}
$$

where $\tilde{\mathbf{n}}$ is given by (12), while $\mathbf{R}_{1 k}$ represents the correlation matrix evaluated for the desired user and the $k$ th interfering user. The correlation matrix $\mathbf{R}_{1 k}$ has the same form as (16), except that the user-related subscript of 2 is now replaced by $k$. 


\section{PERformance Results}

In this section we provide a range of simulation results, in order to illustrate the achievable performance of the SDMA system considered in conjunction with the spatial correlation receiver. As an example, we assumed that in the SDMA system each mobile user was equipped with two transmit antennas, while the BS employed $M=2$ receive antenna arrays, each having $L=2$ array elements. Furthermore, in order to provide an insight into the effects of the multiuser interference as a function of the users' specific spatial signature, we assumed that only $K=2$ users were supported by the SDMA system, where one of them was the desired user and the other one was the interfering user.

Fig. 2 shows the bit error rate (BER) versus signal-to-noise (SNR) per bit, i.e. $E_{b} / N_{0}$ performance of the SDMA system, with respect to the Nakagami fading parameters of $m=$ $0.5,0.8,1.0,1.2,1.5,1.8$ and 2.0 . In this scenario we assumed that the SDMA system supported $K=1$ user. Hence, no multiuser interference was imposed on the desired user As shown in Fig. 2, the BER performance improves, as the fading becomes less severe, i.e. as the Nakagami fading parameter's value increases. According to the results of Fig. 2 we also observe that in the presence of a single interfer, the MIMO based SDMA system is capable of achieving a low BER even in the low SNR per bit range. As shown in Fig. 2, for the worst transmission environment considered, which corresponds to $m=0.5$, a BER of $10^{-3}$ can be achieved at an SNR of $E_{b} / N_{0}=8 \mathrm{~dB}$. By contrast, for the best case considered, namely for $m=2.0$, a BER of $10^{-5}$ may be achieved at an SNR of $E_{b} / N_{0}=6.5 \mathrm{~dB}$. Note that these low BERs are achieved, since the SDMA system is capable of efficiently exploiting the joint benefits of combined transmit diversity, receive diversity as well as receiver beamforming.

In Fig. 3 we show the BER versus SNR per bit performance of the SDMA system considered, when it supports $K=2$ users. In our simulations we assumed that the DOA angles were $\psi_{11}=\psi_{12}=30^{\circ}$ for user 1 and $\psi_{21}=\psi_{22}=60^{\circ}$ for user 2. According to the results of Figs. 3 we observe that as in Fig. 2, the BER performance improves, when increasing the Nakagami fading parameter's value. However, as a consequence of the identical DOA angles of the desired user and the interfering user, aggressive multiuser interference is imposed on the desired signal. Consequently, in contrast to the results of Fig. 2, Fig. 3 demonstrates that the BER performance was significantly degraded and hence relatively high error floors were observed in both of these figures.

In order to demonstrate the effects of various DOAs on the achievable BER performance, Fig. 4 and Fig. 5 show the BER versus the DOA performance of the SDMA system considered. As in Fig. 3, in Fig. 4 we also assumed that the DOAs recorded, when transmitting from a mobile user to different BS receive antenna arrays are perfectly correlated. More quantitatively, for the desired user we assumed that we had $\psi_{11}=\psi_{12}=0^{\circ}$, while for the interfering user we had $\psi_{21}=\psi_{22}$, which assumed angles spanning the range $\left[-180^{\circ}, 180^{\circ}\right]$. In the context of Fig. 5 we assumed that the DOA recorded when transmitting from each of the mobile transmit antennas to each of the BS receive antenna arrays were independent. The results of Fig. 5 were obtained by assuming that we had $\psi_{11}=\psi_{12}=0^{\circ}$, and that both $\psi_{21}$ and $\psi_{22}$ assumed values in the range of $\left[-180^{\circ}, 180^{\circ}\right]$. From the results of Figs. 4 and 5 we observe that when the interfering signals are spatially orthogonal to the desired signal, the multiuser interference can be efficiently mitigated by the BS's receiver with the aid of the associated beamforming applied to the BS's receiver array output signals. Furthermore, the results of Fig. 5 suggest that the BER performance can be significantly improved, provided that the interfering signal is orthogonal to the desired signal at either of the BS's receive antenna arrays. However, when the interfering signals impinging upon both of the BS's receive antenna arrays have similar DOAs to that of the desired users' signals, then the interfering user will impose strong interference on the desired users.

\section{CONCLUSIONS}

In this contribution a SDMA system has been proposed, where each mobile transmitter has two transmit antennas and the BS receiver is constituted by $M$ number of antenna arrays, each having $L$ elements. The performance of the proposed SDMA system has been investigated, when the SDMA signals considered are detected using the so-called spatial correlation detection scheme. The achievable BER performance of the SDMA system has been evaluated, when assuming that the SDMA signals are transmitted over Nakagami- $m$ fading channels. Our simulation results demonstrate that the proposed SDMA system is capable of suppressing the interfering multiuser signals having DOA angles sufficiently different from that of the desired signals. By contrast, strong multiuser interference may be imposed on the desired signals, when these interfering signals arrive from similar directions.

\section{REFERENCES}

[1] J. H. Winters, "On the capacity of radio communication systems with diversity in a Rayleigh fading environment." IEEE Joumal on Selected Areas in Communications, vol. SAC-5, pp. 871-878, June 1987.

[2] I. E. Telatar, "Capacity of multi-antenna Gaussian channels," European Transactions on Telecommunications, vol. 10, pp. 585-595, Nov./Dec. 1999.

[3] A. Goldsmith, S. A. Jafar, N. Jindal, and S. Vishwanath. "Capacity limits of MIMO channels," IEEE Journal on Selected Areas in Communications. vol. 21, pp. 684-702. June 2003.

[4] "Special issue on MIMO systems and applications," IEEE Journal on Selected Areas in Communications, vol. 21, April 2003.

[5] "Special issue on MIMO systems and applications," IEEE Joumal on Selected Areas in Communications, vol. 21, May 2003

[6] S. M. Alamouti, "A simple transmit diversity technique for wireless communications," IEEE Joumal on Selected Areas in Communications, vol. 16, pp. 1451-1458, October 1998.

[7] V. Tarokh, N. Seshadri, and A. R. Calderbank, "Space-time codes for high data rate wireless communication: performance criterion and code construction," IEEE Transactions on Infonnation Theory, vol. 44, pp. 744 765. March 1998.

[8] A. F. Naguib and R. Calderbank, "Space-time coding and signal processing for high data rate wireless communications," Wireless Communications and Mobile Computing, vol. 1, pp. 13-34, January-March 2001

[9] S. Verdu, Multiuser Detection. Cambridge University Press, 1998. 


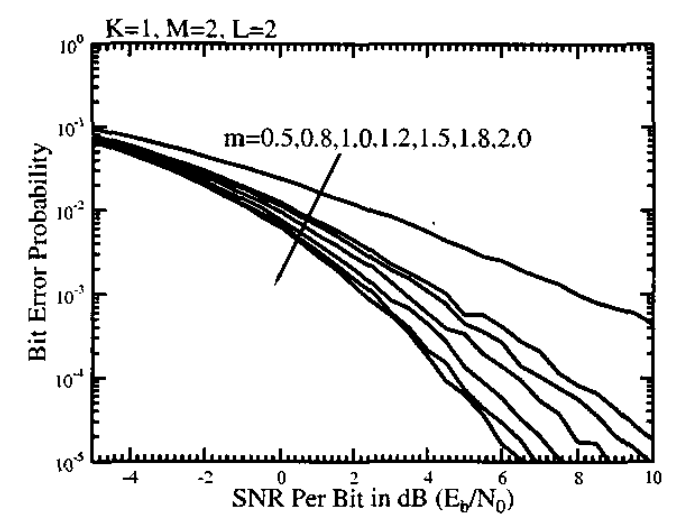

Fig. 2. BER versus SNR per bit performance of the SDMA system using $T x=2$ transmit antennas, $M=2$ receive antenna arrays of each having $L=2$ sensors, and supporting $K=1$ number of user, when communicating over the Nakagami- $m$ fading channels corresponding to the fading parameters of $m=0.5,0.8,1,1.2,1.5,1.8,2.0$

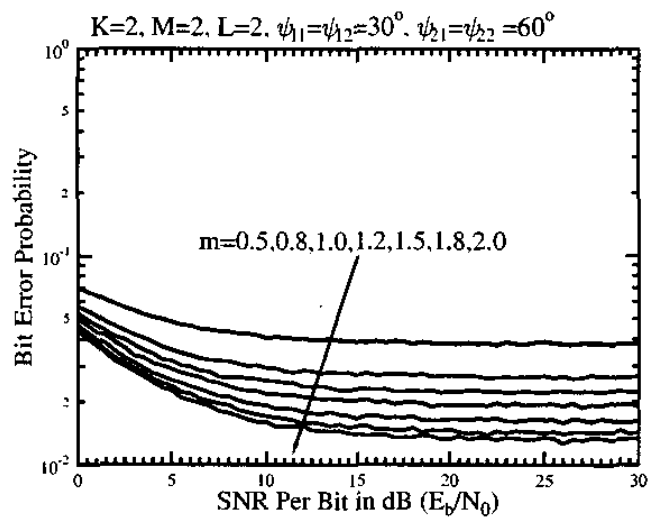

Fig. 3. BER versus SNR per bit performance of the SDMA system using $T x=2$ transmit antennas, $M=2$ receive antenna arrays, each having $L=2$ sensors, and supporting $K=2$ number of user, when communicating over various Nakagami- $m$ fading channels corresponding to the fading parameters of $m=0.5,0.8,1,1.2,1.5,1.8,2.0$. Furthermore, in our simulations we assumed that, for a given user the DOAs recorded at the first and second receive antennas were perfectly correlated, i.e. we assumed $\psi_{11}=\psi_{12}=30^{\circ}$ and $\psi_{21}=\psi_{22}=60^{\circ}$

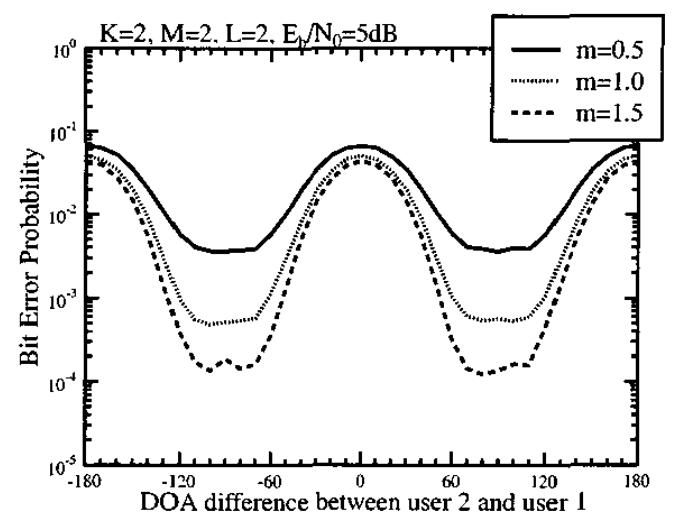

Fig. 4. BER performance of the SDMA system as a function of the directionof-arrival (DOA) angular difference of the interfering signals. In our simulations we assumed that for a given user. the DOAs recorded the first and second receive antennas were perfectly correlated. i.e. we assumed $\psi_{11}=\psi_{12}=0^{\circ}$ and $\psi_{21}=\psi_{22}$. The DOA difference is defined as $\psi_{21}-\psi_{11}$. The other parameters used were $T_{x}=2, M=2, L=2$ and the Nakagami- $m$ fading parameters were $m=0.5,1,1.5$.

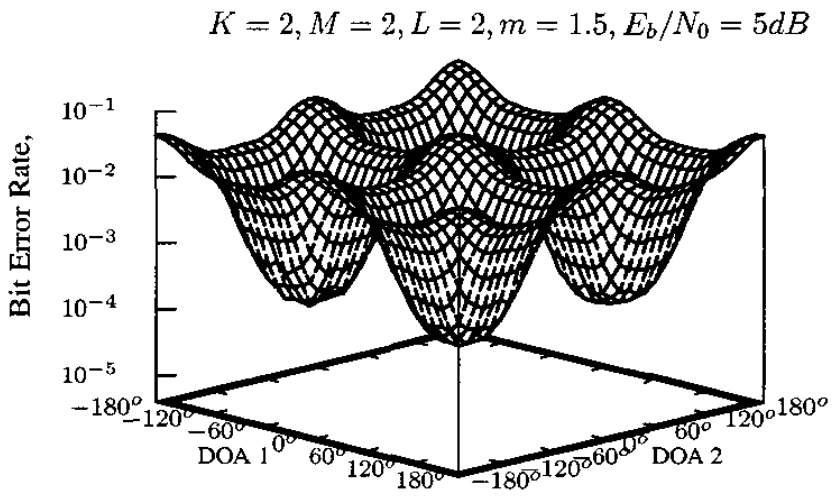

Fig. 5. BER versus DOA performance of the SDMA system using $T x=2$ mobile transmit antennas, $M=2 \mathrm{BS}$ receive antenna arrays each having $L=$ 2 sensors, and supporting $K=2$ users, when communicating over Rayleigh fading channels corresponding to the Nakagami parameter of $m=1$. In this figure DOA 1 and DOA 2 represent the DOA differences between the signals of user 2 and user 1 as received, by the first and second BS antenna arrays respectively. 